\title{
MIXOMA ATRIAL: UM RELATO DE CASO
}

\section{MYXOMA ATRIAL: A CASE REPORT}

\author{
Paula Simone Veiga ${ }^{1^{*}}$, Karin Kristina Bockler ${ }^{2}$, Leandro Ueda ${ }^{3}$, Itamar Regazzo
}

${ }^{1}$ Acadêmica do curso de Medicina do Centro Universitário Assis Gurgacz, ${ }^{2}$ Orientadora, Mestre em Zoologia pela UFPR e docente do curso de Medicina do Centro Universitário Assis Gurgacz, ${ }^{3}$ Médico Cardiologista do Hospital São Lucas, ${ }^{4}$ Médico Intensivista do Hospital São Lucas

*Autor correspondente: paula_veiga10@hotmail.com

DOI: $10.35984 / f j h . v 2 i 1.158$

\section{RESUMO}

Introdução: Os mixomas são tumores cardíacos que em sua grande maioria possuem histogênese benigna e apresentam-se de maneira solitária. Localizam-se em $75 \%$ dos casos do lado esquerdo e são raros, com uma incidência menor que $0,1 \%$ e prevalência em adultos entre a terceira e sexta décadas de vida, predominando em mulheres. Embora a natureza da maioria desses tumores seja de caráter benigno, é importante diagnosticar e iniciar o tratamento com remoção cirúrgica precocemente, pois podem surgir complicações desfavoráveis como acidentes embólicos e levar a mortes súbitas. Objetivo: O propósito deste artigo é analisar um caso clínico de mixoma atrial e seus respectivos exames que estão sob posse do paciente, abordando as manifestações da doença, diagnóstico e tratamento. Com isso, é possível auxiliar em diagnósticos futuros mais precisos, uma vez que a intervenção precoce é imprescindível para vitalidade do paciente.

Palavras-chave: tumores cardíacos. mixoma atrial. histogênese benigna. acidentes embólicos. intervenção precoce.

\begin{abstract}
Introduction: Myxomas are cardiac tumors that mostly have benign histogenesis and present in a solitary manner. They are located in $75 \%$ of cases on the left side and are rare, with an incidence below $0.1 \%$ and prevalence in adults between the third and sixth decades of life, predominating in women. Although the nature of most of these tumors is benign, it is important to diagnose and initiate treatment with early surgical removal, as unfavorable complications such as embolic accidents and sudden death may result. Objective: Through this report, will be analyzed the clinical case of atrial myxoma and their respective exams that are in the possession of the patient, addressing the manifestations of the disease, diagnosis and treatment. With this, more accurate future diagnoses can be assisted, since early intervention is essential for patient vitality.
\end{abstract}

Keywords: heart tumors. atrial myxoma. benign histogenesis. embolic accidents. early intervention.

\section{INTRODUÇÃO}

O envolvimento tumoral do coração tem uma baixa incidência em relação às demais causas de acometimento cardíaco, como doenças de origem anatômica e funcional. No entanto, apesar de ser uma rara doença, existem os tumores cardíacos primários. Dentre eles, conforme os relatórios de patologia, $45 \%$ dos tumores 
benignos do coração são de origem mixomais, podendo ter sido herdados como traço autossômico dominante com mutação do gene supressor de tumor PRKAR1A. Além disso, cerca de $3-10 \%$ dos mixomas podem fazer parte do complexo de Carney, que é uma mutação que desempenha um papel no desenvolvimento de mixomas cardíacos em outras regiões além do átrio. Já os tumores malignos, na maioria dos casos são do tipo sarcoma e apenas 1-2\% são linfomas não-Hodgkin (LESTUZZI, 2016).

Em comparação aos tumores primários, os mixomas são os mais comuns do coração, com histologia benigna em $75 \%$ dos casos. Localizam-se em 75 a $80 \%$ dos casos no átrio esquerdo, $18 \%$ no átrio direito e nos ventrículos ocorrem raramente (PONTES et al, 2011). A prevalência é de adultos entre 30 a 60 anos de vida, ocorrendo mais em mulheres, sendo uma proporção de dois casos no sexo feminino para um em homens. Este tumor é caracterizado por uma anormalidade da proliferação celular das células mesenquimais multipotenciais subendocárdicas, essa proliferação celular forma uma massa revestida por trombos e é recoberta por um estroma mixomatoso, diferenciando-se, histologicamente de trombos organizados, que são recobertos por endotélio (SCHNEIDER et al, 2018).

Apesar de ser uma rara doença, os tumores cardíacos podem apresentar vários sinais e sintomas que a ecocardiografia e outros exames de imagem são capazes de identificar nas lesões. Quando as lesões são encontradas no átrio esquerdo, os sintomas são dispneia, ortopneia, tosse, edema e fadiga e tem como manifestações disso os distúrbios de condução, obstrução e interferência valvar (MIGLIORI et al, 2017).

A síndrome do mixoma é algo raro na transmissão genética familiar, mas mesmo assim deve ser investigada no histórico do paciente. Exames de imagem identificam a lesão que, na maioria das vezes, são pediculadas, amolecidas e com aparência mixóide. A lesão é constituída histologicamente por células mixomatosas, endoteliais, macrófagos e células musculares lisas intervaladas em um estroma mucinóide (SERRANO, 2009).

O limbo da fossa oval é o local preferível de fixação dos mixomas de átrio esquerdo. No entanto, também podem ser encontrados na parede posterior do átrio esquerdo, mas tumores que apresentam essa localização devem ser suspeitados de malignidade. Já os mixomas de átrio direito e de ventrículos são encontrados com menor frequência, e também já foram relatados mixomas de valvas atrioventriculares (BRAUNWALD, 2011).

As manifestações clínicas se apresentam com a tríade de obstrução cardíaca, embolização sistêmica e sintomas constitucionais. A maior ocorrência pelo lado esquerdo gera o prolapso no orifício das válvulas, consequentemente causando a obstrução do influxo ventricular esquerdo e regurgitação mitral. Os episódios embólicos ocorrem em $30 \%$ dos pacientes e os sintomas constitucionais são compostos por mialgia, fraqueza muscular, artralgias, erupção, febre, perda de peso e fadiga. Ao exame físico, podem ser verificados baqueteamento digital e fenômeno de Raynaud (BRAUNWALD, 2011).

O diagnóstico pode ser realizado através da ajuda do eletrocardiograma, podendo apresentar um exame normal ou podem ser registrados distúrbios de condução atrioventriculares com graus de bloqueio, arritmias e alterações da amplitude do complexo QRS. Isso irá depender da invasão ou da localização do tumor. A radiografia simples de tórax também é um exame que pode variar dentro da normalidade ou evidenciar alterações com sinais de congestão, revelando massas 
relacionadas à área cardíaca. No entanto, os melhores exames são os métodos de imagem como ecocardiograma bidimensional, tomografia computadorizada e ressonância magnética, tendo a sensibilidade e especificidade altas para o diagnóstico desta doença (SERRANO, 2009).

O tratamento, devido ao elevado risco de embolização e das manifestações sintomáticas por obstrução intracavitária, é sempre cirúrgico. Além disso, a manipulação da lesão neoplásica deve ser realizada cuidadosamente, visto que pode ocorrer fragmentações causadoras de acidentes embólicos, podendo ser fatal ao paciente. Portanto, é realizado a ressecção completa do tumor, tendo um bom prognóstico com sobrevida de $85 \%$ em 20 anos e risco de recorrência de 1 a $5 \%$ dos casos (PAOLA, 2012).

\section{METODOLOGIA}

O método utilizado no presente artigo caracteriza-se como um relato de caso. A junção de todas as informações foram obtidas por meio da análise documental da evolução do quadro clínico de um paciente atendido no Hospital São Lucas de Cascavel- PR. Foi aprovado pelo Comitê de ética em Pesquisa do Centro Universitário Assis Gurgacz sob o número CAAE: 17321719.9.0000.5219, estando em cumprimento com a Resolução 196/96 do Conselho Nacional de Saúde, visto tratarse de uma pesquisa com ser humano.

\section{RELATO DO CASO}

Paciente do sexo feminino, 37 anos, residente de Dois Vizinhos, encaminhada ao pronto socorro do Hospital São Lucas de Cascavel- PR em 28 de fevereiro de 2019. Relatou queixas de dispneia há algumas semanas associada a mialgia importante em membros inferiores, negou comorbidades e demais queixas. Havia sido investigada pelo reumatologista e sem identificação de irregularidades nesta especialidade. Foi encaminhada ao pneumologista sendo realizado angiotomografia computadorizada e ecocardiograma transtorácico, identificando tromboembolismo pulmonar bilateral subsegmentar com infiltrado alveolar e uma extensa lesão em válvula mitral. A paciente teve admissão na UTI no mesmo dia com suspeita de endocardite e trombose de membros inferiores. Ao consultar o histórico médico da paciente também foi descoberto nódulo pulmonar solitário a direita. No exame físico cardiovascular foi auscultado sopro diastólico e ECG com taquicardia sinusal, ao exame respiratório presença de estertores creptantes difusos, taquidispneia moderada e respiração espontânea com auxílio de $\mathrm{O}_{2}$ em cateter nasal $2 \mathrm{~L} / \mathrm{min}$.

Foram solicitados exames laboratoriais e um ecodopplercardiograma transesofágico, evidenciando presença de grande massa ecogênica dismorfe originária do septo interatrial baixo de bordas e contornos irregulares e mau definidos, medindo aproximadamente em seus maiores diâmetros $80 \mathrm{~mm} \times 4,5 \mathrm{~mm}$, projetando em diástole sobre a cúspide anterior da valva mitral para via de entrada do ventrículo esquerdo, gerando obstrução dinâmica da mesma, com gradiente médio de $8.5 \mathrm{mmHg}$ e em sístole para o átrio esquerdo, gerando refluxo valvar com jato excêntrico moderado, a mesma sugestiva de tumor primário cardíaco-mixoma, conforme a figura 1. Após o resultado dos exames, no dia 01 de fevereiro de 2019 a paciente foi submetida a cirurgia de ressecção transeptal do mixoma gigante. 
O pós operatório imediato da paciente seguiu com choque cardiogênico grave em doses altas de drogas vasoativas, diurese presente, sem sangramento aparente, anasarca e com lactato em elevação, sendo religada a sedação por instabilidade hemodinâmica e feito uso de ventilação mecânica e antibiótico profilático.

A paciente foi avaliada com o exame de ecocardiografia revelando manutenção de grave disfunção de ventrículo esquerdo e veia cava inferior congesta, evoluindo com choque misto (cardiogênico e distributivo). Nos exames laboratoriais apresentouse piora evolutiva da acidose, hiperlactatemia e evoluiu com sepse, sendo optado por implante de balão intra-aórtico para suporte ventricular, altas doses de drogas vasoativas e uso de antibioticoterapia de largo espectro.

No quarto dia de pós operatório foi desligada a sedação, em análise hemodinâmica pela ecocardiografia estimou débito cardíaco de 3,4L/min. No sexto dia apresentou melhora laboratorial, melhora da anasarca e do débito urinário, a paciente também se encontrava menos taquicárdica e com menor dose de inotrópicos, mas no exame de raio- $x$ foi evidenciado atelectasia em base pulmonar direita.

Décimo dia após a cirurgia, a paciente evoluiu com melhora hemodinâmica, tolerando bem o desmame das drogas vasoativas e extubação, melhorando o padrão respiratório em uso de cateter de $\mathrm{O}_{2}$ e diurese efetiva, porém com isquemia de membros inferiores na região anterior dos dois pés. No dia 12 de março a paciente recebeu alta da UTI, mas em seguida apresentou quadro dispneico súbito com cianose, só melhorando após aspiração e inalação. Feito o exame físico, a paciente estava com força muscular diminuída, sem força para expectoração, dificuldade de deglutir líquidos e dispneia devido secreção produtiva orotraqueal, oscilando com episódios de hipossaturação e obstrução de via aérea por secreção. Além disso, ainda apresentava isquemia importante dos membros inferiores e sem melhora.

Após melhora clínica progressiva das intercorrências do pós operatório, dia 25 de abril a paciente pode ser submetida a uma cirurgia vascular para amputação do antepé esquerdo e pododáctilos direito, sem intercorrências. Em 30 de abril recebeu alta hospitalar com sinais vitais estáveis sendo orientada a realizar 0 acompanhamento recorrente com o clínico geral e retorno em 7 dias com 0 cardiologista.

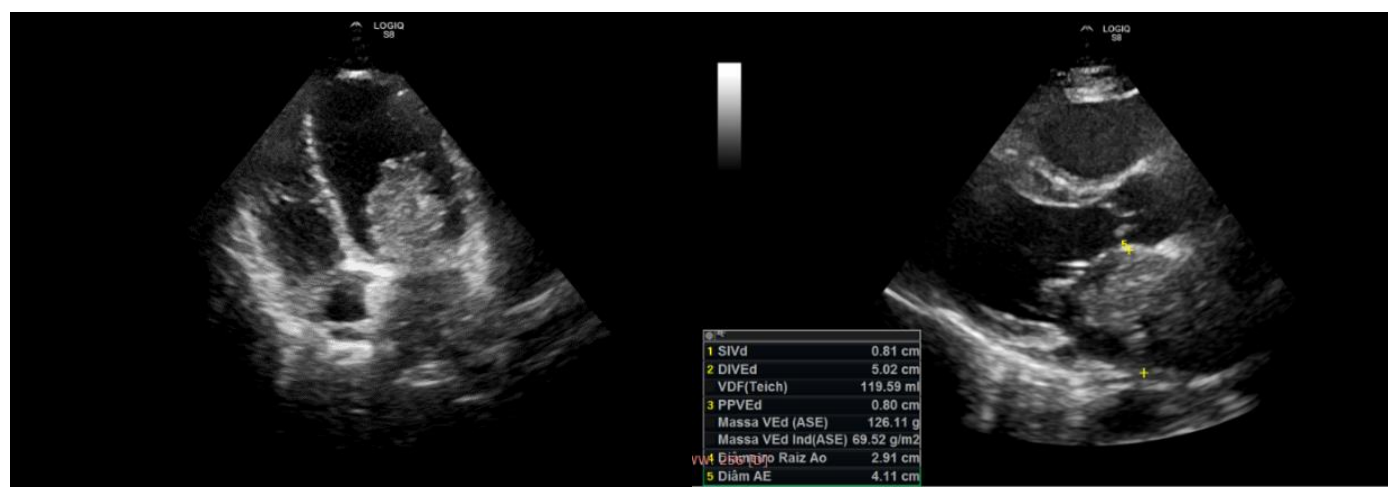

Figura 1 - Ecodopplercardiograma transesofágico, corte apical quatro câmaras e corte longitudinal, respectivamente. Fonte: exame do paciente

\section{DISCUSSÃO}

A abordagem de tratamento mais comum de acordo com a Sociedade Brasileira de Cardiologia é sempre cirúrgica com ressecção completa do tumor em pacientes com mixomas, em razão aos altos riscos de embolização e dos sintomas por FAG Journal of Health - ISSN 2674-550X, 2020, v.2, n.1, p. 162

DOI: $10.35984 / f j h . v 2 i 1.158$ 
obstrução valvar. O prognóstico é favorável a longo prazo, com taxa de sobrevida de $85 \%$ em 20 anos, mas com recidiva de 1 a $5 \%$ dos casos. Portanto, a excisão cirúrgica deve ser planejada assim que o diagnóstico for confirmado, como ocorreu com a paciente no caso descrito. Episódios tromboembólicos são pouco frequentes no pósoperatório e arritmias atriais são as complicações mais comuns após a cirurgia para remoção de mixoma (PAOLA, 2012).

No caso relatado, a paciente teve queixas iniciais apenas de dispneia e ao exame físico sopro diastólico, na qual a pobreza de sinais e sintomas dificultou o diagnóstico clínico suspeitando-se de endocardite, a lesão em válvula mitral e o tromboembolismo pulmonar evidenciados na ecocardiografia transtorácica, são uma das principais complicações da doença fazendo com que chegassem ao diagnóstico, no qual foi confirmado com o ecocardiograma transesofágico, que é o exame considerado de escolha para essa doença quando a ecocardiografia transtorácica não revela resultados conclusivos (PAOLA, 2012).

Apesar da literatura sobre a doença descrever baixa incidência de mortalidade no pós cirúrgico, a paciente obteve complicações graves como o choque misto, a sepse e a isquemia de membros inferiores, ambas contribuíram para maior permanência hospitalar totalizando mais de um mês de internamento. Além disso, a isquemia de membros inferiores que a paciente teve pode ter sido originada como complicação da grande quantidade de drogas vasoativas ou em decorrência do mixoma atrial, sendo uma manifestação rara de embolia periférica, pois de acordo com a literatura, fragmentos tumorais são responsáveis por uma pequena porcentagem dos êmbolos periféricos. Entre os tumores, os de origem cardíaca são os mais associados, sendo os mixomas atriais os mais comuns, relatados em $30 \%$ dos casos (ALCÂNTARA et al, 2012).

\section{CONCLUSÃO}

Embora os mixomas sejam tumores raros, devem ser investigados como diagnóstico diferencial em situações de valvopatias mitral e tricúspide, como foi realizado no caso presente. Apesar de sua histologia ser benigna necessita ser realizada a ressecção tumoral o quanto antes, devido as manifestações da tríade de obstrução clínica, que na paciente se evidenciou em sintomas constitucionais e embólicos como a mialgia, dispneia, o tromboembolismo pulmonar e a isquemia de membros inferiores, respectivamente. No caso relatado, a ecocardiografia transesofágica como exame diagnóstico e o tratamento cirúrgico de ressecção tumoral escolhidos, foram as melhores condutas de acordo com a evolução da doença e a literatura descrita. Portanto, conclui-se que o auxílio de exames de imagem são essenciais para a investigação de algumas doenças e o estudo de casos raros tem importância significativa no diagnóstico preciso precoce na medicina moderna.

\section{REFERÊNCIAS}

ALCÂNTARA, V. Q. M. D. et al. Oclusão arterial aguda de membros inferiores por êmbolo tumoral em paciente com neoplasia de pulmão. J. vasc. bras. vol.11 no.4 Porto Alegre Oct./Dec, 2012. http://dx.doi.org/10.1590/S1677-54492012000400015

BRAUNWALD, E., ZIPES, D.P., ET AL - Braunwald's Heart Disease: A Textbook of Cardiovascular Medicine. Diretrizes da Sociedade Brasileira de Cardiologia. 9th ed, Saunders Elsevier, 2011. 
LESTUZZI, C. Primary tumors of the heart. Current Opinion in Cardiology, 2016. Disponível em https://www.ncbi.nlm.nih.gov/pubmed/27652810, acesso 25.mai, 2019. DOI: 10.1097/HCO.0000000000000335

MIGLIORI, H.R.R; MARQUES B.B; RANGEL, C. M.; OLIVEIRA, J. B de; CARVALHO, A. L. M. M de; RODRIGUES JUNIOR, V. C; CIRQUEIRA, G.V; COSTA, A.A; VERSALI, N.A; MARQUES, B.B; MIGUEZ, F.A; TORRES, F.N. Relato de caso: infarto do miocárdio por mixoma atrial esquerdo. Acta Biomedica Brasiliensia / Volume 8/ $n^{\circ}$ 1/ Julho de 2017. DOI: http://dx.doi.org/10.18571/acbm.133

MOTTA, A. A. R; COLEN FILHO, E.; COLEN, E. A; VIEIRA, J. A. S.; ALVES, M. A.; BORGES, M. F.; ARAÚJO, H. Mixoma do átrio esquerdo: relato de 3 casos. Rev Bras Cir Cardiovascular. São Paulo Oct./Dec, 1997. DOI: 10.1590/S0102-76382008000400022

PAOLA, A. A. V. de; BARBOSA, M. de M.; GUIMARÃES, J. I. Livro texto da Sociedade Brasileira de Cardiologia. Editora Manole, 2012

PONTES, J. C. D. V., SILVA, G. V. R. da, BENFATTI, R. A., \& DUARTE, J. J. Mixoma atrial esquerdo múltiplo. Revista Brasileira de Cirurgia Cardiovascular, 2011. http://dx.doi.org/10.5935/1678-9741.20110030.

SERRANO, C.; TIMERMAN, A.; STEFANINI E. Tratado de Cardiologia SOCESP. $2^{\underline{a}}$ edição. Editora Manole, 2009.

SCHNEIDER, C.; LOPES, M. N. S. C.; LEITE, S. F.; OLIVEIRA, C. C.; SABATOVICZ, N; RESENDE, M. C. Mixoma em Ventrículo Direito. Arq Bras Cardiol, 2018. DOI: $10.5935 / 2318-8219.20180007$ 